Supporting Information

\title{
A Capacitive Sensor Combining Proximity and Pressure Sensing for Accurate Grasping of Prosthetic Hand
}

Chuanyang Ge ${ }^{1 \ddagger}$, Bin Yang ${ }^{2 \ddagger}$, Luyao $\mathrm{Wu}^{1 \ddagger}$, Zhan Duan ${ }^{1}$, Yuyang $\mathrm{Li}^{1}$, Xuanyu Ren ${ }^{1}$, Li Jiang ${ }^{2}$, Jia Zhang ${ }^{1,2 *}$

${ }^{1}$ Key Laboratory of Microsystems and Microstructure Manufacturing, Ministry of Education, Harbin Institute of Technology, No.2 Yikuang Street, Harbin, 150080, China

${ }^{2}$ State Key Laboratory of Robotics and System, Harbin Institute of Technology, No.2 Yikuang Street, Harbin, 150080, China

Corresponding Author: Jia Zhang (zhangjia@hit.edu.cn) 

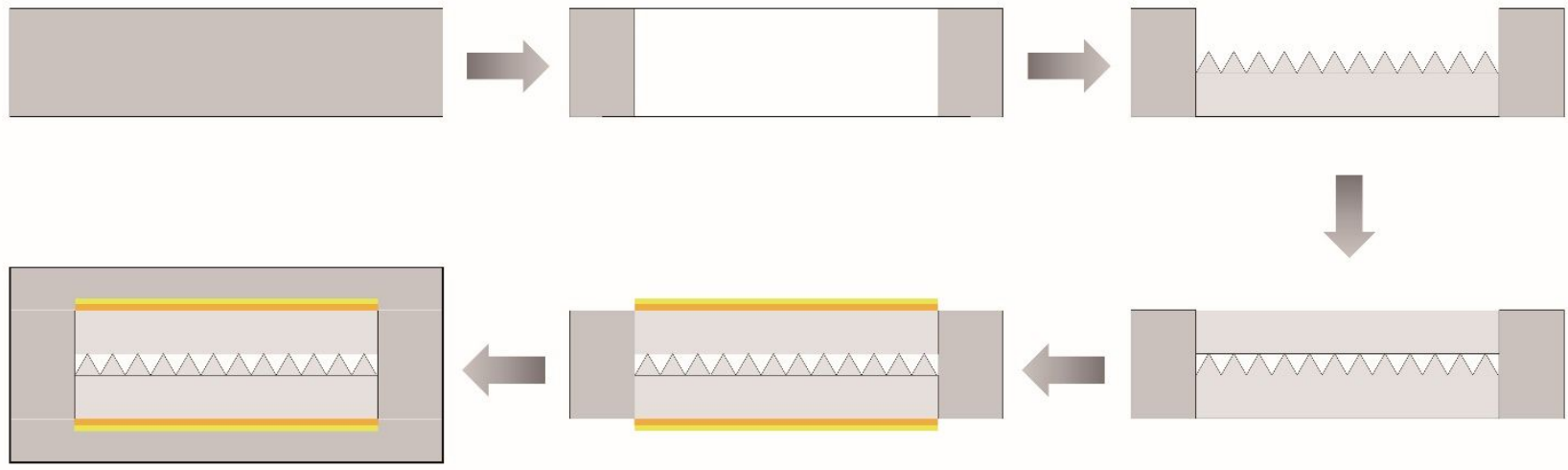

PDMS $\square$ Ecoflex $\square$ Cu electrode $\square$ PI

Figure S1. The synthetic process of capacitive sensor. 


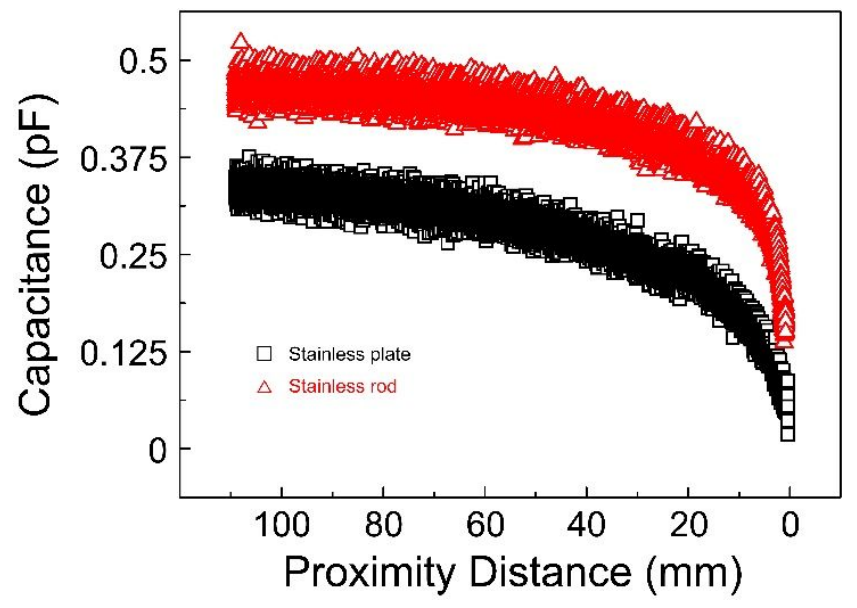

Figure S2. Capacitance change with stainless plate and stainless rod approaching. 

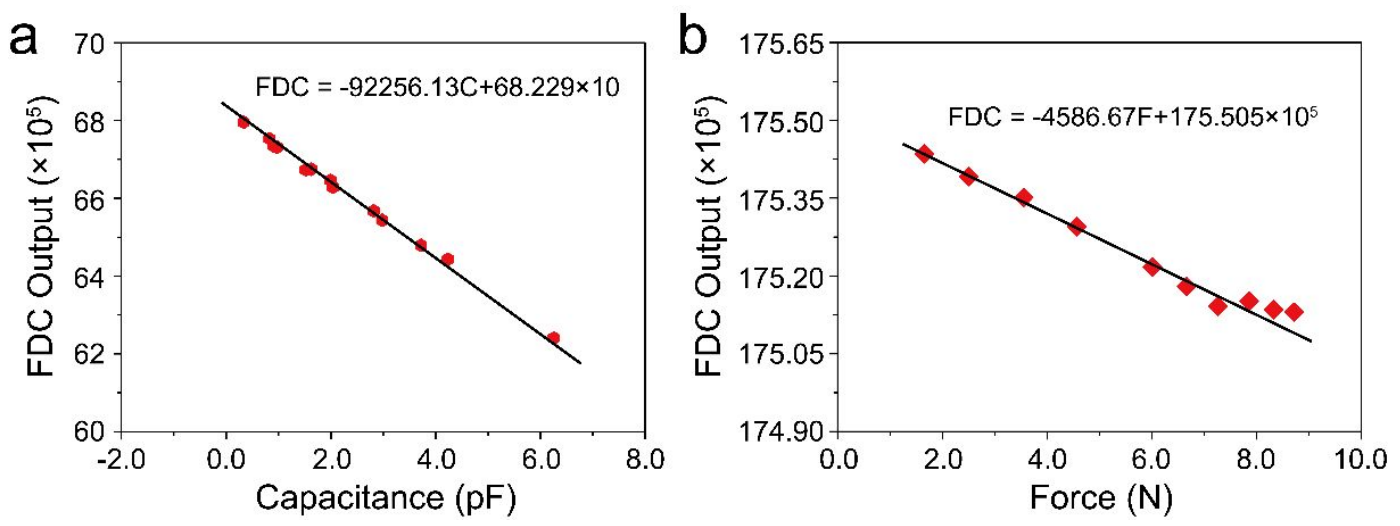

Figure S3. Calibration between FDC output and (a) contact force and (b) capacitance. 